\title{
EFFECT OF DYNAMIC TRAINING PROGRAM DESIGNED BASED ON THE TABATA PROTOCOL ON BALANCE AND STRENGTH PARAMETERS OF ELITE LEVEL COMBAT ATHLETES
}

\author{
İbrahim Orkun Akcan ${ }^{1 \mathrm{i}}$, \\ Cengiz Ölmez ${ }^{2}$, \\ Mehmet Öztaş ${ }^{3}$ \\ ${ }^{1}$ Erzincan Binali Yıldırım University, \\ Faculty of Sports Science, Coaching Education Department, \\ Erzincan Turkey \\ ${ }^{2}$ Ministry of Education, \\ Trabzon Turkey \\ ${ }^{3}$ Erzincan Binali Yıldırım University, \\ Faculty of Sports Science, Sports Management Department, \\ Erzincan Turkey
}

\begin{abstract}
:
The purpose of this study was to investigate the effects of a 6-week dynamic training program designed based on the Tabata protocol on balance and strength parameters of elite level combat athletes. This randomized controlled experimental study with pre-test and post-test was conducted with 25 randomly selected elite level combat athletes who volunteered to participate in the study, 12 in the experimental group ( 5 wrestling, 2 judo, 2 karate, 3 taekwondo) and 13 in the control group. In the pre-test and post-test stages of the study, Biodex Balance SD (Biodex Inc., Shirley, NY) balance device was used to determine static and dynamic balance performances of the athletes. The strength performance of the athletes was determined by measuring back, leg, and grip strength using a back-leg dynamometer (TKK 5402) and a hand grip dynamometer (Takei). After data collection, SPSS 25.0 was used for statistical analysis of the data and the significance level was set at $p<0,05$. At the end of the study, it was determined that the differences between pre-test and post-test body weights and eyes open and closed dynamic balance levels of the experimental group athletes were statistically significant $(p<0,05)$, whereas the differences between the measurement results of control group athletes were not significant $(p>0,05)$. The Tabata protocol is a high intensity interval training technique and is effective on aerobic-anaerobic energy systems. The movement determined for the session is repeated intensely, rhythmically, and continuously during the exercise
\end{abstract}

i Correspondence: email orkunakcan24@gmail.com 
window. As a result, the Tabata protocol supports dynamic balance and strength improvement of elite combat athletes through dynamic exercises.

Keywords: Tabata protocol, strength, balance, performance development

\section{Introduction}

\subsection{Background and Literature Review}

Training is a set of activities that are performed systematically and regularly in order to improve athletic performance. Performance usually refers to carrying out a high-level skill and may require limits to be pushed. For example, activities such as running more than 5000 metres within 14 minutes or completing $21 \mathrm{~km}$ as quickly as possible describe athletic performance (Billat, 2001). The goal here is to be able to complete a particular activity as quickly as possible, or to increase the intensity, volume, or scope of the skills to be performed within a given period of time.

Until today, sports scientists have conducted a lot of research on how athletic performance can be improved in the most effective way and within the shortest amount of time. Such research focuses on selecting the most suitable athlete to achieve the target performance or training the current athlete in the most effective way possible to reach the target performance level (Göksu et al., 2018; Ölmez et al., 2017; Ölmez, 2017; Pion et al., 2014; Reid \& Schneiker, 2008; Weterings et al., 2019). Interval training is a very effective training technique to achieve the target performance.

The principle behind interval training is to re-load the organism while the effects of the previous loading continue and the organism has not yet returned to its normal state by means of resting (Muratlı et al., 2007). Although Foster et al. (2015) reported that steady-state (20 min at $90 \%$ ventilatory threshold) and high intensity interval training (HIIT) programs at submaximal (Meyer et al., 1990) and maximal (Tabata et al., 1996) intensity yielded similar results in terms of performance gains in sedentary participants, it is widely believed that HIIT is highly successful in achieving aerobic and anaerobic gains (Eather et al., 2019; Smith et al., 2009; Jabbour et al., 2017).

When designed using the appropriate method, HIIT provides aerobic endurance athletes such as long distance runners or cyclists with physical and physiological gains in terms of aerobic parameters (Weston et al., 1996; Westgarth-Taylor et al., 1997; Laursen et al., 2002; Lindsay et al., 1996). However, the effects of HIIT on balance and strength development in elite level combat athletes have not been adequately explained and need to be illuminated. In addition, quantitative research shows that training interventions aimed at performance improvement focus on sub-elite level athletes, recreational individuals, or sedentary individuals. This may be due to elite athletes' hesitancy to make changes to their training programs for scientific research purposes (Hawley et al., 1997). Therefore, recommendations made by exercise scientists to coaches and athletes are largely based on anecdotal information from some successful coaches, or training studies conducted with sub-elite level individuals or athletes (Hawley et al., 1997). For this 
reason, this study is quite important to understand the reactions of elite athletes to a training program designed based on the Tabata Protocol, a popular HIIT technique, and to determine its effects on strength and balance improvement.

\section{Material and Methods}

\subsection{Research Pattern}

The study was designed as a pre-test and post-test experimental study with control group (randomized controlled trial) (Figure 1).

\begin{tabular}{|ccccc|}
\hline Group & Pre-test & Activity & Post-test \\
$\mathrm{A} \longrightarrow \mathrm{X} \longrightarrow \mathrm{X} \longrightarrow \mathrm{X}$ \\
$\mathrm{B} \longrightarrow \mathrm{X} \longrightarrow \mathrm{X}$
\end{tabular}

Figure 1: Research pattern

\subsection{Participants}

The study was conducted with 25 randomly selected elite level combat athletes who volunteered to participate in the study, 12 in the experimental group ( 5 wrestling, 2 judo, 2 karate, 3 taekwondo) (mean age $=21,08 \pm 1,17$ years) and 13 in the control group (4 wrestling, 2 judo, 4 karate, 3 taekwondo) (mean age $=21,54 \pm 1,71$ years). All athletes were informed about the study and were explained about the possible benefits and risks of the research. After expression, all athletes were given a written informed consent from which was prepared according to the Declaration of Helsinki. The study was conducted in compliance with the ethical principles of the European Convention and the Helsinki Declaration (ethics principles regarding human experimentation). It was confirmed by the Bioethics Commission of the Gazi University (no: 91610558-302.08.01).

\subsection{Procedure}

Both the experimental and control group athletes participated in their routine training programs specific to their respective branches; however, the experimental group athletes also participated in a 6-week high intensity interval training program designed based on the Tabata protocol 3 days per week in addition to their routine training programs.

Tabata training was performed as 20-second maximum loading and 10-second rest for 8 repetitions. The training program was implemented as sprints, crunches, burpees, plank, mountain climbing, half squats, jumping jacks and push-ups in each training session for 6 weeks. During the 6-week program, the number of sets was increased by one at the end of each week and 1 minute rest time was given between the sets. The athletes warmed up for 15 minutes before the training and cooled down for 10 minutes at the end. 


\begin{tabular}{|c|c|c|c|c|c|}
\hline \multicolumn{6}{|c|}{ Table 1: Tabata training program } \\
\hline Week & Loading & $\begin{array}{c}\text { Rest between } \\
\text { repetitions }\end{array}$ & $\begin{array}{c}\text { Rest between } \\
\text { sets }\end{array}$ & $\begin{array}{l}\text { Number of sets } \\
\text { and repetitions }\end{array}$ & Loading \\
\hline $\begin{array}{l}\text { 1st } \\
\text { Week }\end{array}$ & $\begin{array}{c}20 \\
\text { seconds }\end{array}$ & $\begin{array}{c}10 \\
\text { seconds }\end{array}$ & \multirow{6}{*}{$1 \mathrm{~min}$} & $\begin{array}{c}4 \text { sets, } \\
8 \text { repetitions }\end{array}$ & \multirow{6}{*}{$\begin{array}{c}\text { Maximal } \\
\text { (all out } \\
\text { effort) }\end{array}$} \\
\hline $\begin{array}{l}\text { 2nd } \\
\text { Week }\end{array}$ & $\begin{array}{c}20 \\
\text { seconds }\end{array}$ & $\begin{array}{c}10 \\
\text { seconds }\end{array}$ & & $\begin{array}{c}4 \text { sets, } \\
8 \text { repetitions }\end{array}$ & \\
\hline $\begin{array}{l}\text { 3rd } \\
\text { Week }\end{array}$ & $\begin{array}{c}20 \\
\text { seconds }\end{array}$ & $\begin{array}{c}10 \\
\text { seconds }\end{array}$ & & $\begin{array}{c}5 \text { sets, } \\
8 \text { repetitions }\end{array}$ & \\
\hline $\begin{array}{l}\text { th } \\
\text { Week }\end{array}$ & $\begin{array}{c}20 \\
\text { seconds }\end{array}$ & $\begin{array}{c}10 \\
\text { seconds }\end{array}$ & & $\begin{array}{c}5 \text { sets, } \\
8 \text { repetitions }\end{array}$ & \\
\hline $\begin{array}{l}\text { 5th } \\
\text { Week }\end{array}$ & $\begin{array}{c}20 \\
\text { seconds }\end{array}$ & $\begin{array}{c}10 \\
\text { seconds }\end{array}$ & & $\begin{array}{c}6 \text { sets, } \\
8 \text { repetitions }\end{array}$ & \\
\hline $\begin{array}{l}\text { 6th } \\
\text { Week }\end{array}$ & $\begin{array}{c}20 \\
\text { seconds }\end{array}$ & $\begin{array}{c}10 \\
\text { seconds }\end{array}$ & & $\begin{array}{c}6 \text { sets, } \\
8 \text { repetitions }\end{array}$ & \\
\hline
\end{tabular}

\subsection{Body Composition Measurements}

In order to determine the body composition of the athletes, their height, body weight, body mass index, body fat percentage, and body fat mass values were determined.

The height of the athletes was measured with a Holtain brand (UK) stadiometer, while body mass index, body fat percentage, and total body fat measurements were performed using the foot-to-foot bioelectrical impedance analysis (Tanta Body Composition Analyzer) at 9.00 AM.

\subsection{Determination of Balance Performance}

The dynamic and static balance performance with eyes open and closed were determined using a Biodex brand (Biodex, Inc., Shirley, New York) balance system. This system has a moving balance platform that can tilt the surface up to $20^{\circ}$, has a $360^{\circ}$ joint motion span, and is equipped with a monitor which shows the user as a representative dot. The screen of the Biodex balance system is divided into 4 zones and 4 quadrants, showing various directions and degrees of difficulty (Figure 1).

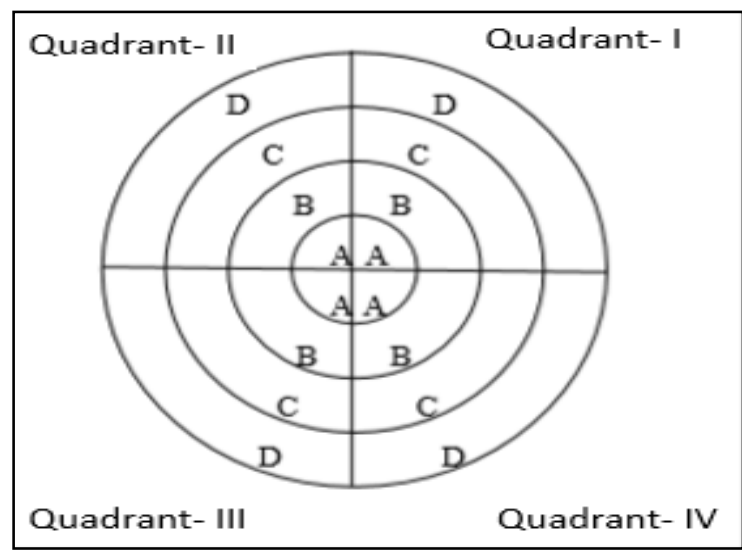

Figure 1: Screenshot of the Biodex balance system's screen during implementation 
Balance zone A: $0^{\circ}-5^{\circ}$ incline

Balance zone B: $6^{\circ}-10^{\circ}$ incline

Balance zone C: $11^{\circ}-15^{\circ}$ incline

Balance zone D: $16^{\circ}-20^{\circ}$ incline 1st Quadrant: right-front $90^{\circ}$ zone

2nd Quadrant: left-front $90^{\circ}$ zone

3rd Quadrant: left-rear $90^{\circ}$ zone

4th Quadrant: right-rear $90^{\circ}$ zone

\subsection{Implementation of the Test}

The athletes were allowed to perform 2 trials before the actual implementation in order for them to adapt to the balance system. The athletes were allowed to rest for 20 seconds between the trials, and the actual implementation was initiated. 3 actual measurements were made and the best performance was used in the assessment. Overall balance index, anterior-posterior (forward-backward) balance index and medial-lateral (inwardoutward) balance index results were obtained with the measurement. The implementation was carried out in level 8 mode with a duration of 30 seconds and rest intervals of 15 seconds.

\subsection{Determination of Strength Performance}

Grip strength: The grip strength was measured using an isometric dynamometer (TakeiHand Grip) with athletes standing upright and an angle of approximately $45^{\circ}$ between the arm and the body. The athletes used their dominant hand (Heyward, 2002).

Back strength: A back and leg (Takei-Back \& Lift) dynamometer was used for measuring the back strength. After placing their feet on the dynamometer's stand with their knees and arms stretched, their backs straight, and their torso in a slightly forward tilted position, the athletes pulled the dynamometer bar vertically upwards with full strength to perform the measurement. The athletes were reminded to pull the bar trying to get their backs into extension position, not their arms or legs (Heyward, 2002).

Leg strength: A back and leg (Takei-Back \& Lift) dynamometer was used for measuring the leg strength. For leg strength measurements, the athletes were asked to place their feet on the dynamometer stand with their knees bent at an angle of $130^{\circ}-140^{\circ}$, to hold their arms stretched, their backs straight, and their bodies slightly forward tilted, and then to pull the dynamometer bar vertically while trying to bring their legs to the extension position. Athletes were reminded to pull the bar using their legs without using their backs and arms during the measurement (Heyward, 2002). All strength measurements were repeated 3 times and the best results were recorded in $\mathrm{kg}$.

\subsection{Statistical Analysis}

The normal distribution of the data was controlled before the analysis using the ShapiroWilk normality test. The Paired Samples T-Test was used for parametric data and the Wilcoxon test was used for non-parametric data within the groups, while the Independent Samples T-Test was used for parametric data and the Mann Whitney U Test was used for non-parametric data between the groups. Parametric data were presented as mean and standard deviation, and non-parametric data with median values. SPSS 25 was used for statistical analysis and the level of significance was set at $p<0,05$. 


\section{Results and Discussion}

The athletes' body composition values and balance (eyes closed-open, dynamic-static) and strength (hand grip, back, leg) performance results were determined with in-group and inter-group measurements.

Table 2: Body composition values of the athletes

\begin{tabular}{|c|c|c|c|c|c|c|c|c|c|}
\hline \multirow[t]{2}{*}{ Variables } & \multirow[t]{2}{*}{ Groups } & \multicolumn{3}{|c|}{$\begin{array}{c}\text { 1st } \\
\text { Measurement }\end{array}$} & \multicolumn{3}{|c|}{$\begin{array}{c}\text { 2nd } \\
\text { Measurement }\end{array}$} & \multicolumn{2}{|c|}{$\begin{array}{l}\text { 1st measurement } x \\
\text { 2nd measurement }\end{array}$} \\
\hline & & mean & Sd. & $\mathrm{p}$ & mean & Sd. & p & Change (\%) & $p$ \\
\hline \multirow{2}{*}{ Body Weight (kg) } & EG $(n=12)$ & 70,53 & 5,60 & \multirow{2}{*}{0,663} & 71,41 & 5,58 & \multirow{2}{*}{0,786} & 1,24 & $0,033^{*}$ \\
\hline & $C G(n=13)$ & 72,42 & 6,62 & & 72,61 & 6,82 & & 0,25 & 0,506 \\
\hline \multirow{2}{*}{ Height (cm) } & $\mathrm{EG}(\mathrm{n}=12)$ & 174,58 & 5,04 & \multirow{2}{*}{0,219} & 174,58 & 5,04 & \multirow{2}{*}{0,219} & - & - \\
\hline & $\mathrm{CG}(\mathrm{n}=13)$ & 177,31 & 5,68 & & 177,31 & 5,68 & & - & - \\
\hline \multirow{2}{*}{ BMI (kg/m2) } & $\mathrm{EG}(\mathrm{n}=12)$ & 23,58 & 1,18 & \multirow{2}{*}{0,369} & 23,58 & 1,50 & \multirow{2}{*}{0,253} & $-0,04$ & 0,156 \\
\hline & CG $(n=13)$ & 23,12 & 1,59 & & 23,15 & 1,64 & & 0,13 & 0,620 \\
\hline \multirow{2}{*}{ Body Fat (\%) } & EG $(n=12)$ & 11,69 & 2,71 & \multirow{2}{*}{0,957} & 10,59 & 3,47 & \multirow{2}{*}{0,703} & $-9,41$ & 0,182 \\
\hline & CG $(n=13)$ & 11,74 & 4,23 & & 10,87 & 3,97 & & $-7,40$ & 0,196 \\
\hline \multirow{2}{*}{ Fat Mass } & EG $(n=12)$ & 8,28 & 1,86 & \multirow{2}{*}{0,892} & 7,48 & 2,25 & \multirow{2}{*}{0,430} & $-9,76$ & 0,209 \\
\hline & $\mathrm{CG}(\mathrm{n}=13)$ & 8,59 & 3,38 & & 8,05 & 3,36 & & $-6,36$ & 0,221 \\
\hline
\end{tabular}

$\mathrm{BMI}=$ Body Mass Index; ${ }^{*} \mathrm{p}<0,05$

According to the 1st measurement results related to body compositions of the athletes, there was no significant difference between the control group and experimental group in terms of body weight, height, body mass index, body fat percentage, and total fat mass ( $p>0,05)$. According to the 2 nd measurement results, there was a significant difference between the control group and experimental group in terms of body weight $(\mathrm{p}>0,05)$, whereas no significant difference was found in terms of height, body mass index, body fat percentage, and total fat mass $(\mathrm{p}>0,05)$ (Table 2$)$.

The examination of the dynamic balance performance of the athletes with eyes closed showed that there was no significant difference between the control group and the experimental group in any of the dimensions measured in the 1st and the 2nd measurement ( $p>0,05)$. The 1nd and 2nd intra-group measurements, on the other hand, showed that while there was no significant difference between the 1st and 2nd measurements of the control group athletes, a significant difference was found between the 1st and 2nd measurements of the experimental group athletes in terms of overall balance, medial-lateral balance (inward and outward swing), balance zone A $\left(0^{\circ}-5^{\circ}\right.$ incline), and balance zone $\mathrm{B}\left(6^{\circ}-10^{\circ}\right.$ incline) $(\mathrm{p}<0,05)$ (Table 3$)$. 
Table 3: Results of the eyes closed-dynamic balance measurements

\begin{tabular}{|c|c|c|c|c|c|c|c|c|c|}
\hline \multirow{2}{*}{ Variables } & \multirow{2}{*}{ Groups } & \multicolumn{3}{|c|}{$\begin{array}{c}\text { 1st } \\
\text { Measurement }\end{array}$} & \multicolumn{3}{|c|}{$\begin{array}{c}\text { 2nd } \\
\text { Measurement }\end{array}$} & \multicolumn{2}{|c|}{$\begin{array}{l}\text { 1st measurement } x \\
\text { 2nd measurement }\end{array}$} \\
\hline & & $\begin{array}{l}\text { Mean/ } \\
\text { median }\end{array}$ & Sd. & p & $\begin{array}{l}\text { Mean/ } \\
\text { median }\end{array}$ & Sd. & $\mathbf{p}$ & Change (\%) & $\mathrm{p}$ \\
\hline \multirow{2}{*}{$\begin{array}{l}\text { Overall } \\
\text { balance }\end{array}$} & $\mathrm{EG}(\mathrm{n}=12)$ & 5,52 & 1,50 & \multirow{2}{*}{0,870} & 11,13 & & \multirow{2}{*}{0,132} & $-19,79$ & 0,028 \\
\hline & CG $(n=13)$ & 6,01 & 2,38 & & 14,73 & & & $-8,58$ & 0,421 \\
\hline \multirow{2}{*}{$\begin{array}{l}\text { Anterior- } \\
\text { Posterior }\end{array}$} & $\mathrm{EG}(\mathrm{n}=12)$ & 3,78 & 1,44 & \multirow{2}{*}{0,605} & 11,38 & & \multirow{2}{*}{0,224} & $-16,74$ & 0,125 \\
\hline & $C G(r$ & 4,06 & 1,56 & & 14,50 & & & $-4,92$ & 0,666 \\
\hline \multirow{2}{*}{$\begin{array}{l}\text { Medial- } \\
\text { Lateral }\end{array}$} & EG $(n=12)$ & 3,13 & 1,06 & \multirow{2}{*}{0,785} & 42 & 0,87 & \multirow{2}{*}{0,075} & $-22,67$ & 0,016 \\
\hline & CG $(n=13)$ & 3,56 & 1.73 & & 3,09 & 0,93 & & -13.17 & 0,363 \\
\hline \multirow{2}{*}{$\begin{array}{l}\text { Balance zone } \\
\text { A (\% sec.) }\end{array}$} & EG $(n=$ & 48,92 & 20,04 & \multirow{2}{*}{0,892} & 65,75 & 14,97 & \multirow{2}{*}{0,167} & & 0,008 \\
\hline & CG $(n=13)$ & 49,54 & 23,57 & & 56,69 & 16,62 & & 14, & 0,208 \\
\hline \multirow{2}{*}{$\begin{array}{l}\text { Balance zone } \\
\text { B (\% sec.) }\end{array}$} & EG $(n=12)$ & 41,83 & 17,18 & \multirow{2}{*}{0,210} & 12,96 & & \multirow{2}{*}{0,380} & $-32,67$ & 0,009 \\
\hline & CG $(n=13)$ & 34,69 & 12,76 & & 13,04 & & & $-9,53$ & 0,462 \\
\hline \multirow{2}{*}{$\begin{array}{l}\text { Balance zone } \\
\text { C (\% sec.) }\end{array}$} & EG (n & & 7,03 & \multirow{2}{*}{0,412} & 4,25 & 3,98 & \multirow{2}{*}{0,308} & $-35,44$ & 0,085 \\
\hline & CG $(n=13)$ & 9,08 & 8,30 & & 6,15 & 5,03 & & $-32,20$ & 0,168 \\
\hline \multirow{2}{*}{$\begin{array}{l}\text { Balance zone } \\
\text { D (\% sec.) }\end{array}$} & EG $(n=12)$ & 11,67 & & \multirow{2}{*}{0,326} & 11,50 & & \multirow{2}{*}{0,184} & $-31,25$ & 0,752 \\
\hline & CG $(n=13)$ & 14,23 & & & 14,38 & & & $-13,79$ & 0,799 \\
\hline \multirow{2}{*}{$\begin{array}{l}\text { 1st quadrant } \\
\text { (\% sec.) }\end{array}$} & EG $(n=12)$ & 12,96 & & \multirow{2}{*}{0,978} & 13,88 & & \multirow{2}{*}{0,682} & 15,03 & 0,638 \\
\hline & CG $(n=13)$ & 13,04 & & & 12,19 & & & 9,76 & 0,889 \\
\hline \multirow{2}{*}{$\begin{array}{l}\text { 2nd quadrant } \\
\text { (\% sec.) }\end{array}$} & $\mathrm{EG}(\mathrm{n}=12)$ & 33,08 & 22,54 & \multirow{2}{*}{0,892} & 13,42 & & \multirow{2}{*}{0,931} & $-29,97$ & 0,224 \\
\hline & CG $(n=13)$ & 31,15 & 19,31 & & 12,62 & & & $-27,65$ & 0,173 \\
\hline & EG $(n=12)$ & 21,25 & 20,79 & & 24,58 & 13,93 & & 15,69 & 0,432 \\
\hline & CG $(n=13)$ & 26,46 & 14,76 & & 28,08 & 21,77 & 0,041 & 6,10 & 0,724 \\
\hline 4th quadrant & EG $(n=12)$ & 12,58 & & & 13,29 & & & 13,74 & 0,386 \\
\hline & CG $(n=13)$ & 13,38 & & 0,18 & 12,73 & & 0,96 & 23,86 & 0,442 \\
\hline
\end{tabular}

${ }^{*} \mathrm{p}<0,05 ;{ }^{* *} \mathrm{p}<0,01$

The examination of the dynamic balance performance of the athletes with eyes open showed that there was no significant difference between the control group and the experimental group in any of the dimensions measured in the 1st and the 2nd measurement ( $p>0,05)$. The 1nd and 2nd intra-group measurements, on the other hand, showed that while there was no significant difference between the 1st and 2nd measurements of the control group athletes, a significant difference was found between the 1st and 2nd measurements of the experimental group athletes in terms of overall balance, anterior-posterior balance (forward-backward swing), medial-lateral balance (inward and outward swing), 2nd quadrant, and 4th quadrant $(\mathrm{p}<0,05)$ (Table 4). 
Table 4: Results of the eyes open-dynamic balance measurements

\begin{tabular}{|c|c|c|c|c|c|c|c|c|c|}
\hline \multirow{2}{*}{ Variables } & \multirow{2}{*}{ Groups } & \multicolumn{3}{|c|}{$\begin{array}{c}\text { 1st } \\
\text { Measurement }\end{array}$} & \multicolumn{3}{|c|}{$\begin{array}{c}\text { 2nd } \\
\text { Measurement }\end{array}$} & \multicolumn{2}{|c|}{$\begin{array}{l}\text { 1st measurement } x \\
\text { 2nd measurement }\end{array}$} \\
\hline & & $\begin{array}{l}\text { Mean/ } \\
\text { median }\end{array}$ & Sd. & $\mathrm{p}$ & $\begin{array}{l}\text { Mean/ } \\
\text { median }\end{array}$ & Sd. & $\mathrm{p}$ & Change (\%) & p \\
\hline \multirow{2}{*}{$\begin{array}{l}\text { Overall } \\
\text { balance }\end{array}$} & $\mathrm{EG}(\mathrm{n}=12)$ & 13,63 & - & \multirow{2}{*}{0,683} & 0,97 & 0,67 & \multirow{2}{*}{0,421} & $-45,54$ & 0,006 \\
\hline & $C G(n=13)$ & 12,42 & - & & 1,22 & 0,83 & & $-17,28$ & 0,271 \\
\hline \multirow{2}{*}{$\begin{array}{l}\text { Anterior- } \\
\text { Posterior }\end{array}$} & $\mathrm{EG}(\mathrm{n}=12)$ & 13,63 & - & \multirow{2}{*}{0,682} & 12,63 & - & \multirow{2}{*}{0,966} & $-40,54$ & 0,023 \\
\hline & CG $(n=13)$ & 12,42 & - & & 13,35 & - & & $-30,37$ & 0,069 \\
\hline \multirow{2}{*}{$\begin{array}{l}\text { Medial- } \\
\text { Lateral }\end{array}$} & $E G(n=12)$ & 13,83 & - & \multirow{2}{*}{0,585} & 0,38 & 0,35 & \multirow{2}{*}{0,109} & $-63,78$ & 0,006 \\
\hline & CG $(n=13)$ & 12,23 & - & & 0,65 & 0,43 & & $-22,04$ & 0,122 \\
\hline \multirow{2}{*}{$\begin{array}{l}\text { Balance zone } \\
\text { A (\% sec.) }\end{array}$} & EG $(n=12)$ & 13,00 & - & \multirow{2}{*}{1,000} & 14,00 & - & \multirow{2}{*}{0,276} & 0,08 & 0,317 \\
\hline & CG $(n=13)$ & 13,00 & - & & 12,08 & - & & $-8,47$ & 0,285 \\
\hline \multirow{2}{*}{$\begin{array}{l}\text { Balance zone } \\
\text { B (\% sec.) }\end{array}$} & EG $(n=12)$ & 13,00 & - & \multirow{2}{*}{1,000} & 12,00 & - & \multirow{2}{*}{0,276} & $-100,00$ & 0,317 \\
\hline & CG $(n=13)$ & 13,00 & - & & 13,92 & - & & 2750,00 & 0,285 \\
\hline \multirow{2}{*}{$\begin{array}{l}\text { Balance zone } \\
\text { C (\% sec.) }\end{array}$} & $\mathrm{EG}(\mathrm{n}=12)$ & 0,00 & 0,00 & \multirow{2}{*}{1,000} & 0,00 & 0,00 & \multirow{2}{*}{ - } & 0,00 & 1,000 \\
\hline & CG $(n=13)$ & 0,00 & 0,00 & & 0,00 & 0,00 & & 0,00 & 1,000 \\
\hline \multirow{2}{*}{$\begin{array}{l}\text { Balance zone } \\
\text { D (\% sec.) }\end{array}$} & EG $(n=12)$ & 0,00 & 0,00 & \multirow{2}{*}{1,000} & 0,00 & 0,00 & \multirow{2}{*}{ - } & 0,00 & 1,000 \\
\hline & CG $(n=13)$ & 0,00 & 0,00 & & 0,00 & 0,00 & & 0,00 & 1,000 \\
\hline \multirow{2}{*}{$\begin{array}{l}\text { 1st quadrant } \\
\text { (\% sec.) }\end{array}$} & EG $(n=12)$ & 29,42 & 23,82 & \multirow{2}{*}{0,369} & 12,63 & - & \multirow{2}{*}{0,889} & 11,05 & 0,959 \\
\hline & CG $(n=13)$ & 37,92 & 25,19 & & 13,35 & - & & $-8,52$ & 0,834 \\
\hline \multirow{2}{*}{$\begin{array}{l}\text { 2nd quadrant } \\
\text { (\% sec.) }\end{array}$} & EG $(n=12)$ & 37,75 & 27,45 & \multirow{2}{*}{0,414} & 11,42 & - & \multirow{2}{*}{0,290} & $-74,17$ & 0,003 \\
\hline & CG $(n=13)$ & 27,69 & 17,24 & & 14,46 & - & & $-34,44$ & 0,101 \\
\hline 3rd quadrant & EG $(n=12)$ & 12,67 & - & 827 & 12,75 & - & 08 & 33,47 & 0,824 \\
\hline (\% sec.) & $C G(n=13)$ & 13,31 & - & $0,0<7$ & 13,23 & - & 0,090 & 75,26 & 0,092 \\
\hline 4th quadrant & $\mathrm{EG}(\mathrm{n}=12)$ & 11,67 & - & 0382 & 14,88 & - & 0387 & 144,30 & 0,019 \\
\hline (\% sec.) & CG $(n=13)$ & 14,23 & - & 0,382 & 11,27 & - & 0,387 & 8,95 & 0,937 \\
\hline
\end{tabular}

${ }^{*} \mathrm{p}<0,05 ;{ }^{* *} \mathrm{p}<0,01$

The examination of the dynamic balance performance of the athletes with eyes open showed that there was no significant difference between the control group and the experimental group in any of the dimensions measured in the 1 st measurement $(p>0,05)$, whereas a significant difference was found in the 2nd measurement in terms of mediallateral balance in favor of the experimental group $(p<0,05)$. The 1 nd and 2 nd intra-group measurements showed that there was a significant difference between the 1st and second measurements of the control group and the experimental group athletes in terms of 3rd quadrant and 4th quadrant $(\mathrm{p}<0,05)$ (Table 5). 
Table 5: Results of the eyes open-static balance measurements

\begin{tabular}{|c|c|c|c|c|c|c|c|}
\hline \multirow[t]{2}{*}{ Variables } & \multirow[t]{2}{*}{ Groups } & \multicolumn{2}{|c|}{$\begin{array}{c}\text { 1st } \\
\text { Measurement }\end{array}$} & \multicolumn{2}{|c|}{$\begin{array}{c}\text { 2nd } \\
\text { Measurement }\end{array}$} & \multicolumn{2}{|c|}{$\begin{array}{l}\text { 1st measurement } x \\
\text { 2nd measurement }\end{array}$} \\
\hline & & Median & $\mathrm{p}$ & Median & p & Change (\%) & p \\
\hline \multirow{2}{*}{$\begin{array}{l}\text { Overall } \\
\text { balance }\end{array}$} & EG $(n=12)$ & 13,04 & \multirow{2}{*}{0,977} & 13,42 & \multirow{2}{*}{0,489} & $-33,94$ & 0,528 \\
\hline & CG $(n=13)$ & 12,96 & & 12,62 & & $-49,88$ & 0,104 \\
\hline \multirow{2}{*}{$\begin{array}{l}\text { Anterior- } \\
\text { Posterior }\end{array}$} & $E G(n=12)$ & 13,00 & \multirow{2}{*}{1,000} & 13,00 & \multirow{2}{*}{-} & 0,00 & 1,000 \\
\hline & CG $(n=13)$ & 13,00 & & 13,00 & & 0,00 & 1,000 \\
\hline \multirow{2}{*}{$\begin{array}{l}\text { Medial- } \\
\text { Lateral }\end{array}$} & $\mathrm{EG}(\mathrm{n}=12)$ & 13,04 & \multirow{2}{*}{0,977} & 13,42 & \multirow{2}{*}{0,489} & $-33,94$ & 0,528 \\
\hline & CG $(n=13)$ & 12,96 & & 12,62 & & $-49,88$ & 0,104 \\
\hline \multirow{2}{*}{$\begin{array}{l}\text { Balance zone } \\
\text { A (\% sec.) }\end{array}$} & EG $(n=12)$ & 12,54 & \multirow{2}{*}{0,744} & 12,58 & \multirow{2}{*}{0,492} & 23,76 & 0,458 \\
\hline & CG $(n=13)$ & 13,42 & & 13,38 & & 22,16 & 0,144 \\
\hline \multirow{2}{*}{$\begin{array}{l}\text { Balance zone } \\
\text { B (\% sec.) }\end{array}$} & EG $(n=12)$ & 13,00 & \multirow{2}{*}{1,000} & 13,00 & \multirow{2}{*}{ - } & 0,00 & 1,000 \\
\hline & CG $(n=13)$ & 13,00 & & 13,00 & & 0,00 & 1,000 \\
\hline \multirow{2}{*}{$\begin{array}{l}\text { Balance zone } \\
\text { C (\% sec.) }\end{array}$} & EG $(n=12)$ & 13,54 & \multirow{2}{*}{0,298} & 13,00 & \multirow{2}{*}{-} & $-100,00$ & 0,317 \\
\hline & CG $(n=13)$ & 12,50 & & 13,00 & & 0,00 & 1,000 \\
\hline \multirow{2}{*}{$\begin{array}{l}\text { Balance zone } \\
\text { D (\% sec.) }\end{array}$} & EG $(n=12)$ & 13,29 & \multirow{2}{*}{0,834} & 13,42 & \multirow{2}{*}{0,492} & $-33,94$ & 0,458 \\
\hline & CG $(n=13)$ & 12,73 & & 12,62 & & $-49,63$ & 0,144 \\
\hline \multirow{2}{*}{$\begin{array}{l}\text { 1st quadrant } \\
\text { (\% sec.) }\end{array}$} & EG $(n=12)$ & 13,00 & \multirow{2}{*}{1,000} & 13,00 & \multirow[t]{2}{*}{-} & 0,00 & 1,000 \\
\hline & CG $(n=13)$ & 13,00 & & 13,00 & & 0,00 & 1,000 \\
\hline \multirow{2}{*}{$\begin{array}{l}\text { 2nd quadrant } \\
\text { (\% sec.) }\end{array}$} & $\mathrm{EG}(\mathrm{n}=12)$ & 13,00 & \multirow{2}{*}{1,000} & 13,00 & \multirow{2}{*}{-} & 0,00 & 1,000 \\
\hline & CG $(n=13)$ & 13,00 & & 13,00 & & 0,00 & 1,000 \\
\hline \multirow{2}{*}{$\begin{array}{l}\text { 3rd quadrant } \\
\text { (\% sec.) }\end{array}$} & EG $(n=12)$ & 13,75 & 0580 & 14,63 & 0135 & $-87,87$ & 0,046 \\
\hline & CG $(n=13)$ & 12,31 & 0,580 & 11,50 & 0,135 & $-99,99$ & 0,043 \\
\hline 4th quadrant & EG $(n=12)$ & 12,25 & 050 & 11,38 & 0135 & 34,45 & 0,046 \\
\hline (\% sec.) & CG $(n=13)$ & 13,69 & 0,080 & 14,50 & 0,135 & 15,04 & 0,043 \\
\hline
\end{tabular}

${ }^{*} \mathrm{p}<0,05 ;{ }^{* *} \mathrm{p}<0,01$

The examination of the static balance performance of the athletes with eyes closed showed that there was no significant difference between the control group and the experimental group in any of the dimensions measured in the 1st and the 2nd measurement $(p>0,05)$. The 1nd and 2nd intra-group measurements showed that there was no significant difference between the 1st and 2nd measurements of the control group athletes, whereas a significant difference was found for the experimental group athletes in terms of 3rd quadrant and 4th quadrant $(p<0,05)$ (Table 6). 
Table 6: Results of the eyes closed-static balance measurements

\begin{tabular}{|c|c|c|c|c|c|c|c|}
\hline \multirow[t]{2}{*}{ Variables } & \multirow[t]{2}{*}{ Groups } & \multicolumn{2}{|c|}{$\begin{array}{c}\text { 1st } \\
\text { Measurement }\end{array}$} & \multicolumn{2}{|c|}{$\begin{array}{c}\text { 2nd } \\
\text { Measurement }\end{array}$} & \multicolumn{2}{|c|}{$\begin{array}{l}\text { 1st measurement } x \\
\text { 2nd measurement }\end{array}$} \\
\hline & & Median & $\mathrm{p}$ & Median & $\mathrm{p}$ & Change (\%) & $\mathrm{p}$ \\
\hline \multirow{2}{*}{$\begin{array}{l}\text { Overall } \\
\text { balance }\end{array}$} & EG $(n=12)$ & 15,42 & \multirow{2}{*}{0,106} & 12,67 & \multirow{2}{*}{0,843} & $-53,56$ & 0,137 \\
\hline & CG $(n=13)$ & 10,77 & & 13,31 & & $-23,20$ & 0,779 \\
\hline \multirow{2}{*}{$\begin{array}{l}\text { Anterior- } \\
\text { Posterior }\end{array}$} & $E G(n=12)$ & 13,00 & \multirow{2}{*}{1,000} & 13,00 & \multirow{2}{*}{-} & 0,00 & 1,000 \\
\hline & CG $(n=13)$ & 13,00 & & 13,00 & & 0,00 & 1,000 \\
\hline \multirow{2}{*}{$\begin{array}{l}\text { Medial- } \\
\text { Lateral }\end{array}$} & $\mathrm{EG}(\mathrm{n}=12)$ & 15,42 & \multirow{2}{*}{0,106} & 12,67 & \multirow{2}{*}{0,843} & $-53,56$ & 0,137 \\
\hline & CG $(n=13)$ & 10,77 & & 13,31 & & $-23,20$ & 0,779 \\
\hline \multirow{2}{*}{$\begin{array}{l}\text { Balance zone } \\
\text { A (\% sec.) }\end{array}$} & EG $(n=12)$ & 10,54 & \multirow{2}{*}{0,100} & 13,00 & \multirow{2}{*}{0,840} & 66,42 & 0,137 \\
\hline & CG $(n=13)$ & 15,27 & & 13,00 & & 10,00 & 0,672 \\
\hline \multirow{2}{*}{$\begin{array}{l}\text { Balance zone } \\
\text { B (\% sec.) }\end{array}$} & EG $(n=12)$ & 13,50 & \multirow{2}{*}{0,563} & 13,04 & \multirow{2}{*}{0,955} & $-66,67$ & 0,414 \\
\hline & CG $(n=13)$ & 12,54 & & 12,96 & & $-85,71$ & 0,317 \\
\hline \multirow{2}{*}{$\begin{array}{l}\text { Balance zone } \\
\text { C (\% sec.) }\end{array}$} & EG $(n=12)$ & 13,00 & \multirow{2}{*}{1,000} & 13,04 & \multirow{2}{*}{0,955} & 0,00 & 1,000 \\
\hline & CG $(n=13)$ & 13,00 & & 12,96 & & $-66,67$ & 0,317 \\
\hline \multirow{2}{*}{$\begin{array}{l}\text { Balance zone } \\
\text { D (\% sec.) }\end{array}$} & EG $(n=12)$ & 15,00 & \multirow{2}{*}{0,172} & 12,96 & \multirow{2}{*}{0,840} & $-45,84$ & 0,205 \\
\hline & CG $(n=13)$ & 11,15 & & 13,04 & & $-13,86$ & 0,933 \\
\hline \multirow{2}{*}{$\begin{array}{l}\text { 1st quadrant } \\
\text { (\% sec.) }\end{array}$} & EG $(n=12)$ & 13,00 & \multirow{2}{*}{1,000} & 13,00 & \multirow[t]{2}{*}{-} & 0,00 & 1,000 \\
\hline & CG $(n=13)$ & 13,00 & & 13,00 & & 0,00 & 1,000 \\
\hline \multirow{2}{*}{$\begin{array}{l}\text { 2nd quadrant } \\
\text { (\% sec.) }\end{array}$} & $\mathrm{EG}(\mathrm{n}=12)$ & 13,00 & \multirow{2}{*}{1,000} & 13,00 & \multirow{2}{*}{-} & 0,00 & 1,000 \\
\hline & CG $(n=13)$ & 13,00 & & 13,00 & & 0,00 & 1,000 \\
\hline \multirow{2}{*}{$\begin{array}{l}\text { 3rd quadrant } \\
\text { (\% sec.) }\end{array}$} & EG $(n=12)$ & 14,13 & 0443 & 13,54 & 0433 & $-96,11$ & 0,028 \\
\hline & CG $(n=13)$ & 11,96 & 0,443 & 12,50 & 0,433 & $-65,55$ & 0,091 \\
\hline 4th quadrant & EG $(n=12)$ & 11,88 & 0443 & 12,46 & 0432 & 41,19 & 0,028 \\
\hline (\% sec.) & CG $(n=13)$ & 14,04 & 0,443 & 13,50 & 0,433 & 14,69 & 0,091 \\
\hline
\end{tabular}

${ }^{*} \mathrm{p}<0,05 ;{ }^{* *} \mathrm{p}<0,01$

The examination of the strength balance performance of the athletes showed that there was no significant difference between the control group and the experimental group in any of the dimensions measured in the 1st measurement ( $p>0,05)$, whereas a significant difference was found in the 2nd measurement in terms of back strength in favor of the experimental group $(\mathrm{p}<0,05)$. The 1nd and 2 nd intra-group strength measurements showed that there was no significant difference between the 1st and 2nd measurements of the control group and the experimental group athletes $(p<0,05)($ Table 7$)$. 


\begin{tabular}{|c|c|c|c|c|c|c|c|c|c|}
\hline \multicolumn{10}{|c|}{ Table 7: Strength performance values } \\
\hline \multirow[t]{2}{*}{ Variables } & \multirow[t]{2}{*}{ Groups } & \multicolumn{3}{|c|}{$\begin{array}{c}\text { 1st } \\
\text { Measurement }\end{array}$} & \multicolumn{3}{|c|}{$\begin{array}{c}\text { 2nd } \\
\text { Measurement }\end{array}$} & \multicolumn{2}{|c|}{$\begin{array}{l}\text { 1st measurement } x \\
\text { 2nd measurement }\end{array}$} \\
\hline & & Mean & Sd. & $\mathbf{p}$ & Mean & Sd. & $\mathrm{p}$ & Change $(\%)$ & $\mathrm{p}$ \\
\hline \multirow{2}{*}{$\begin{array}{l}\text { Back strength } \\
(\mathrm{kg})\end{array}$} & $\begin{array}{l}\text { EG } \\
(n=12)\end{array}$ & 143,03 & 17,76 & \multirow{2}{*}{0,092} & 151,15 & 20,31 & \multirow{2}{*}{$0,006^{*}$} & 5,67 & 0,182 \\
\hline & $\begin{array}{l}\text { CG } \\
(\mathrm{n}=13)\end{array}$ & 130,98 & 26,01 & & 130,62 & 12,23 & & $-0,28$ & 0,600 \\
\hline \multirow{2}{*}{$\begin{array}{l}\text { Leg strength } \\
(\mathrm{kg})\end{array}$} & $\begin{array}{l}\mathrm{EG} \\
(\mathrm{n}=12)\end{array}$ & 134,34 & 26,04 & \multirow{2}{*}{0,550} & 143,26 & 16,87 & \multirow{2}{*}{0,703} & 6,64 & 0,158 \\
\hline & $\begin{array}{l}\text { CG } \\
(\mathrm{n}=13)\end{array}$ & 129,49 & 26,76 & & 139,07 & 18,12 & & 7,40 & 0,152 \\
\hline \multirow{2}{*}{$\begin{array}{l}\text { Grip strength- } \\
\text { right }(\mathrm{kg})\end{array}$} & $\begin{array}{l}\mathrm{EG} \\
(\mathrm{n}=12)\end{array}$ & 44,24 & 8,54 & \multirow{2}{*}{0,463} & 48,84 & 10,10 & \multirow{2}{*}{0,550} & 10,41 & 0,071 \\
\hline & $\begin{array}{l}\text { CG } \\
(\mathrm{n}=13)\end{array}$ & 42,70 & 7,08 & & 47,36 & 9,00 & & 10,93 & 0,101 \\
\hline \multirow{2}{*}{$\begin{array}{l}\text { Grip strength- } \\
\text { left }(\mathrm{kg})\end{array}$} & $\begin{array}{l}\text { EG } \\
(n=12)\end{array}$ & 44,20 & 6,89 & \multirow{2}{*}{0,231} & 44,68 & 9,48 & \multirow{2}{*}{0,957} & 1,08 & 0,754 \\
\hline & $\begin{array}{l}\text { CG } \\
(\mathrm{n}=13)\end{array}$ & 41,27 & 4,13 & & 44,96 & 8,48 & & 8,96 & 0,116 \\
\hline
\end{tabular}

${ }^{*} \mathrm{p}<0,05$

\section{Recommendations}

Similar studies with different groups and with athletes at different levels, from different sexes and age groups need to be conducted in order to obtain clearer findings about the effects of Tabata training on athletic improvement. In addition, the implementation of Tabata training over a longer period of time may yield different results related to the athletic gains.

\section{Conclusion}

The purpose of this study was to investigate the effects of a 6-week dynamic training program designed based on the Tabata protocol on balance and strength parameters of elite level combat athletes. To this end, the balance and strength performances of 25 athletes from wrestling, judo, karate, and taekwondo were examined before and after the 6-week program.

The data collected for the control and experimental group athletes before the experimental stage showed that there was no significant difference between the groups in terms of body composition, balance, and strength, that the control and experimental group athletes had similar body compositions and balance and strength performances (Table 2-7).

The body composition results of the experimental and control group athletes at the end of the 6-week training program showed that there was no significant difference between the groups according to the 2nd measurement results; however, a significant 
difference was found between the intra-group body weight measurements of the experimental group athletes (Table 2). In spite of a $9.41 \%$ decrease in the body fat percentages of the experimental group athletes, their body weight increased by $1.24 \%$, which may be explained by increased muscle hypertrophy caused by the dynamic training program designed based on the Tabata protocol. The fact that a strength improvement was observed for the athletes at the end of the 6-week period, albeit not statistically significant, strengthens the likelihood of muscle hypertrophy (Table 7). It has been known for many years that dynamic and static strength exercises cause skeletal muscle hypertrophy (Rasch \& Morehouse, 1957).

The examination of the changes in the dynamic balance performance of the athletes with their eyes closed showed that the experimental group athletes had a significant improvement in overall balance by $19.79 \%$ and in medial-lateral (inwardoutward) balance by $22.67 \%$. The significant changes in the A and B balance zones of the athletes indicated that the balance performance of the experimental group athletes in the 2nd measurements shifted from zone A $\left(0^{\circ}-5^{\circ}\right.$ incline) to zone $\mathrm{B}\left(6^{\circ}-10^{\circ}\right.$ incline). This suggests that the experimental group athletes were able to maintain their balance in a more difficult zone (Table 3).

The examination of the changes in the dynamic balance performance of the athletes with their eyes open showed that the experimental group athletes had a significant improvement in overall balance by $45.54 \%$, in anterior-posterior (forwardbackward) balance by $40.54 \%$, in medial-lateral (inward-outward) balance by $63.78 \%$ (Table 4).

The examination of the changes in the static balance performances of the athletes showed that there was no significant difference between the intra-group and inter-group performance results with eyes open and closed (Table 5-6). The training parameters designed based on the Tabata protocol consisted of dynamic exercises, which did not contribute to the improvement of static balance, but contributed significantly to the improvement of dynamic balance performance. Studies in the literature report the contribution of dynamic exercises to dynamic balance improvement (Kamide et al., 2009; Forsberg et al., 2016; Mohammadi et al., 2012).

The examination of the performance changes in the strength parameters of the athletes as a result of the dynamic exercises designed based on the Tabata protocol showed that the difference between the improvement rates of the experimental and control group athletes in terms of back strength was significant, and no significant intragroup or inter-group difference was found in terms of other parameters (Table 7). In terms of back strength performance, it can be said that while the experimental group athletes achieved $5.67 \%$ improvement at the end of 6 weeks, the control group athletes did not show any improvement. This difference between the groups in terms of back strength performances may have been caused by Tabata-based dynamic exercises performed by the experimental group athletes. The fact that the experimental and control group athletes exhibited similar improvement in other strength parameters, but the experimental group athletes displayed higher improvement in terms of back strength 
supports this idea. The improvement in grip and leg strength was similar, but the improvement in back strength was different between the groups, which may be due to the training program. This suggests that athletes should pay more attention to exercises that improve back strength in training sessions. It can be said that dynamic exercises designed based on the Tabata protocol eliminated the experimental group's lack of back strength-improving exercises.

The improvement of an athletic skill or performance occurs rapidly in novice athletes, but it is much slower in elite athletes. This is due to the fact that elite athletes are closer to their peak than novices. The present study was conducted with athletes from wrestling, karate, judo, and taekwondo, for which strength and balance performance are highly important. Although the athletes were at the elite level in their respective fields, dynamic exercise groups designed based on the Tabata protocol were found to contribute to the dynamic balance and strength performance of the athletes at various rates. The fact that there was no improvement in static balance, hand and leg strength performance indicates that the type and nature of the exercises that make up the Tabata training should be selected carefully. In conclusion, purposefully planned Tabata training contributes to the balance and strength improvement of athletes.

\section{Conflict of Interest Statement}

The authors declare no conflicts of interests.

\section{About the Authors}

İbrahim Orkun Akcan, Physical Education Teacher at Turkish Military School (20092019), Assistant Professor at Erzincan Binali Yıldırım University, Turkey. (2021- ...), Kickboxing Coach, orcid.org/0000-0001-6983-2145

Cengiz Ölmez, PhD, Physical Education Teacher at Ministry of Education, Trabzon University, Turkey. Taekwondo Coach. orcid.org/0000-0001-8584-6272

Mehmet Öztaş, Lecturer at Erzincan Binali Yıldırım University, Turkey. (2014-...) orcid.org/0000-0003-4689-6490

\section{References}

Billat, L. V. (2001). Interval training for performance: A scientific and empirical practice. Special recommendations for middle- and long-distance running. Part I: Aerobic interval training. Sports Medicine, 31(1), 13-31. https://doi.org/10.2165/00007256200131010-00002

Eather, N., Riley, N., Miller, A., Smith, V., Poole, A., Vincze, L., Morgan, P. J., \& Lubans, D. R. (2019). Efficacy and feasibility of HIIT training for university students: The Uni-HIIT RCT. Journal of Science and Medicine in Sport, 22(5), 596-601. https://doi.org/10.1016/j.jsams.2018.11.016 
Forsberg, A., von Koch, L., \& Nilsagård, Y. (2016). Effects on balance and walking with the coduse balance exercise program in people with multiple sclerosis: a multicenter randomized controlled trial. Multiple Sclerosis International, 2016, 7076265. https://doi.org/10.1155/2016/7076265

Foster, C., Farland, C. V, Guidotti, F., Harbin, M., Roberts, B., Schuette, J., Tuuri, A., Doberstein, S. T., \& Porcari, J. P. (2015). The effects of high intensity interval training vs steady state training on aerobic and anaerobic capacity. Journal of Sports Science and Medicine, 14, 747-755. http://www.jssm.org

Göksu, Ö. C., Yüksek, S., \& Ölmez, C. (2018). The Investigation of the Motor Skills of "U” Kategories Soccer Players Who Have Recreative Involvement in Other Sports. Journal of Education and Training Studies, 6(2), 10-17. https://doi.org/10.11114/jets.v6i2.2824

Hawley, J. A., Myburgh, K. H., Noakes, T. D., \& Dennis, S. C. (1997). Training techniques to improve fatigue resistance and enhance endurance performance. Journal of Sports Sciences, 15(3), 325-333. https://doi.org/10.1080/026404197367335

Heyward, V. (2002). Advanced Fitness Assessment \& Exercise Prescription (4th ed.). Human Kinetics. https://www.amazon.com/Advanced-Assessment-Prescription-4thPublishers-Hardcover/dp/B00M3V74QM

Jabbour, G., Iancu, H.-D., Mauriège, P., Joanisse, D. R., \& Martin, L. J. (2017). Highintensity interval training improves performance in young and older individuals by increasing mechanical efficiency. Physiological Reports, 5(7), e13232. https://doi.org/10.14814/phy2.13232

Kamide, N., Shiba, Y., \& Shibata, H. (2009). Effects on balance, falls, and bone mineral density of a home-based exercise program without home visits in communitydwelling elderly women: a randomized controlled trial. Journal of Physiological Anthropology, 28(3), 115-122. https://doi.org/10.2114/jpa2.28.115

Laursen, P. B., Blanchard, M. A., \& Jenkins, D. G. (2002). Acute High-Intensity Interval Training Improves $\mathrm{T}_{\mathrm{vent}}$ and Peak Power Output in Highly Trained Males. Canadian Journal of Applied Physiology, 27(4), 336-348. https://doi.org/10.1139/h02019

Lindsay, F. H., Hawley, J. A., Myburgh, K. H., Schomer, H. H., Noakes, T. D., \& Dennis, S. C. (1996). Improved athletic performance in highly trained cyclists after interval training. Medicine and Science in Sports and Exercise, 28(11), 1427-1434. https://doi.org/10.1097/00005768-199611000-00013

Meyer, K., Lehmann, M., Sünder, G., Keul, J., \& Weidemann, H. (1990). Interval versus continuous exercise training after coronary bypass surgery: A comparison of training-induced acute reactions with respect to the effectiveness of the exercise methods. Clinical Cardiology, 13(12), 851-861. https://doi.org/10.1002/clc.4960131208

Mohammadi, V., Alizadeh, M., \& Gaieni, A. (2012). The effects of six weeks strength exercises on static and dynamic balance of young male athletes. Procedia - Social and Behavioral Sciences, 31, 247-250. https://doi.org/10.1016/j.sbspro.2011.12.050 
Muratlı, S., Kalyoncu, O., \& Şahin, G. (2007). Antrenman ve müsabaka. Ladin Matbaası.

Ölmez, C. (2017). Müzikli ritim çalışmaları ile yapılan taekwondo ĕğitiminin çocukların teknik ve motorik gelişimlerine etkisi. Karadeniz Teknik Üniversitesi.

Ölmez, C., Yüksek, S., Üçüncü, M., \& Ayan, V. (2017). The relationship between some anthropometric features and 50 meter freestyle swimming performance in 8-12 ages children. Turkiye Klinikleri, 9(3), 95-100. https://doi.org/10.5336/sportsci.201654026

Pion, J., Fransen, J., Lenoir, M., \& Segers, V. (2014). The value of non-sport-specific characteristics for talent orientation in young male judo, karate and taekwondo athletes. Archives of Budo, 10(1), 154. www.archbudo.com

Rasch, P. J., \& Morehouse, L. E. (1957). Effect of static and dynamic exercises on muscular strength and hypertrophy. Journal of Applied Physiology, 11(1), 29-34. https://doi.org/10.1152/jappl.1957.11.1.29

Reid, M., \& Schneiker, K. (2008). Strength and conditioning in tennis: Current research and practice. Journal of Science and Medicine in Sport, 11(3), 248-256. https://doi.org/10.1016/j.jsams.2007.05.002

Smith, A. E., Walter, A. A., Graef, J. L., Kendall, K. L., Moon, J. R., Lockwood, C. M., Fukuda, D. H., Beck, T. W., Cramer, J. T., \& Stout, J. R. (2009). Effects of $\beta$-alanine supplementation and high-intensity interval training on endurance performance and body composition in men; a double-blind trial. Journal of the International Society of Sports Nutrition, 6(1), 1-9. https://doi.org/10.1186/1550-2783-6-5

Tabata, I., Nishimura, K., Kouzaki, M., Hirai, Y., Ogita, F., Miyachi, M., \& Yamamoto, K. (1996). Effects of moderate-intensity endurance and high-intensity intermittent training on anaerobic capacity and $\mathrm{VO}(2 \mathrm{max})$. Medicine and Science in Sports and Exercise, 28(10), 1327-1330. https://doi.org/10.1097/00005768-199610000-00018

Westgarth-Taylor, C., Hawley, J. A., Rickard, S., Myburgh, K. H., Noakes, T. D., \& Dennis, S. C. (1997). Metabolic and performance adaptations to interval training in endurance-trained cyclists. European Journal of Applied Physiology and Occupational Physiology, 75(4), 298-304. https://doi.org/10.1007/s004210050164

Weston, A. R., Myburgh, K. H., Lindsay, F. H., Dennis, S. C., Noakes, T. D., \& Hawley, J. A. (1996). Skeletal muscle buffering capacity and endurance performance after high-intensity interval training by well-trained cyclists. European Journal of Applied $\begin{array}{llll}\text { Physiology and } \quad \text { Occupational } & \text { 75ysiology, }\end{array}$ https://doi.org/10.1007/s004210050119

Weterings, S., Oppewal, A., Eeden, F. M. M., \& Hilgenkamp, T. I. M. (2019). A resistance exercise set for a total body workout for adults with intellectual disabilities, a pilot study. Journal of Applied Research in Intellectual Disabilities, 32(3), 730-736. https://doi.org/10.1111/jar.12552 

be applied to their work. Under the terms of this license, no permission is required from the author(s) or publisher for members of the community to copy, distribute, transmit or adapt the article content, providing a proper, prominent and unambiguous attribution to the authors in a manner that makes clear that the materials are being reused under permission of a Creative Commons License. Views, opinions and conclusions expressed in this research article are views, opinions and conclusions of the author(s). Open Access Publishing Group and European Journal of Physical Education and Sport Science shall not be responsible or answerable for any loss, damage or liability caused in relation to/arising out of conflict of interests, copyright violations and inappropriate or inaccurate use of any kind content related or integrated on the research work. All the published works are meeting the Open Access Publishing requirements and can be freely accessed, shared, modified, distributed and used in educational, commercial and non-commercial purposes under a Creative Commons attribution 4.0 International License (CC BY 4.0). 